\title{
The use of intravenous pulse-steroid therapy in optic neuropathy from nasopharyngeal carcinoma
}

\author{
Karen B. Reyes, ${ }^{1,2}$ Kong Yong Goh ${ }^{1 *}$ \\ 'Department of Ophthalmology, Neuro-Ophthalmology Service, Tan Tock Seng \\ Hospital, Singapore; \\ ${ }^{2}$ Department of Ophthalmology, Neuro-Ophthalmology service, Cardinal Santos \\ Medical Center, Philippines
}

\begin{abstract}
Although nasopharyngeal carcinoma (NPC) is commonly seen in patients from Asia, optic neuropathy is a rare initial presenting symptom of this disease. This is an interventional case report discussing the clinical response of NPC-related optic neuropathy to pulse-steroid therapy.

We discuss two patients who initially presented with signs of optic neuropathy. On imaging, a skull-base tumor was noted infiltrating the area of the affected optic nerve. An excision biopsy of the tumor revealed nasopharyngeal carcinoma. Both patients were pulsed with intravenous (IV) methlyprednisolone one $\mathrm{gm} /$ day for three days, followed by oral steroids (one $\mathrm{mg} / \mathrm{kg} / \mathrm{BW}$ ) with gradual tapering. After two weeks, both showed visual improvement and chemo-and radiation therapy commenced at this time.

In these two cases, pulse-steroid therapy did not hinder the chemo- and radiation therapy for nasopharyngeal carcinoma. Steroid therapy brought immediate visual recovery and gradual oral tapering could assist in optimizing visual outcomes for patients with compressive optic neuropathy from nasopharyngeal carcinoma.
\end{abstract}

Key words: Optic neuropathy; nasopharyngeal carcinoma; IV pulse-steroid therapy; vision loss; skull-base tumor

\section{Introduction}

Nasopharyngeal carcinoma (NPC) commonly affects patients from the Asian region (Southern China, Taiwan, Singapore) $)^{1-4}$ and optic neuropathy is a rare initial presenting symptom of this disease. ${ }^{3-7}$ We discuss two cases of patients who initially presented with signs of optic neuropathy. On imaging, a skull-base tumor was noted infiltrating the area of the affected optic nerve. Both patients were pulsed with IV methlyprednisolone one $\mathrm{gm} / \mathrm{day}$ for three days followed by oral steroids (one $\mathrm{mg} / \mathrm{kg}$ body weight) with gradual tapering over the course of two months. Approximately after the second week of steroid therapy, both patients showed visual improvement. However, the clinical course of the two patients differed after a month, as the second case was non-compliant to the steroid therapy.

A retrospective review of all cases diagnosed with NPC who initially presented with optic neuropathy at Tan Tock Seng Hospital, Singapore during the period of January 2000-January 2010 was done. All case notes with the diagnosis of both NPC and optic

*The authors have no financial interests regarding the products mentioned in this paper.

Correspondence: Karen Bulan Reyes, MD, Cardinal Santos Medical Center, 10 Wilson St, Greenhills, San Juan, Philippines 1500.

E-mail:kurr_b_reyes@yahoo.com 
neuropathy were searched in the main patient database of the hospital searching through the ENT, ophthalmology and neuro-surgery services.

Only two cases fulfilled the criteria of having both diagnosis of NPC and optic neuropathy. Both cases underwent a thorough neuro-ophthalmologic examination (visual acuity, Ishihara color plates, and visual-field testing; anterior and posterior eye evaluation, extra-ocular muscle motility examination and cranial nerves assessment). In addition, skull base neuro-imaging was done to establish presence of a mass lesion in the area and excision biopsy by the ears, nose and throat (ENT) service to confirm diagnosis of NPC. Each case was reviewed for demographic data, clinical course before and after diagnosis and treatment.

\section{Case reports}

\section{Case 1}

A 57-year-old Chinese man presented a three-month history of blurred vision in the right eye with associated headaches. He also had suffered from epistaxis and left tinnitus for a year with a loss of appetite and weight loss. On examination, visual acuity (VA) was hand movements on the right eye with an afferent pupillary defect and mild inferior optic-disc swelling. The left-eye vision and optic disc were normal. However, on motility exam, there was a limitation of abduction in the left eye. Corneal sensations were decreased in both eyes. A CT-scan of the orbits and brain showed a mass with bony erosions in the skull base with extension to the pons, and a high suspicion of involvement of the right optic nerve (Fig. 1a). He was immediately referred to ENT where he underwent excision biopsy.

While awaiting the biopsy results, he was immediately started on IV pulse methylprednisolone one gram/day for three days. Histology revealed that he had an undifferentiated

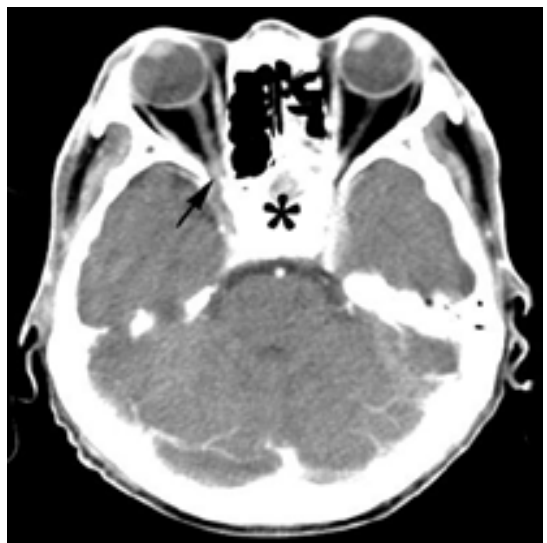

Fig. 1a. Case 1. Axial contrast-enhanced CT scan of the head showing a mass lesion at the post-nasal space with extension to the pons (asterisk). The posterior portion of the right optic nerve was thickened (arrow).

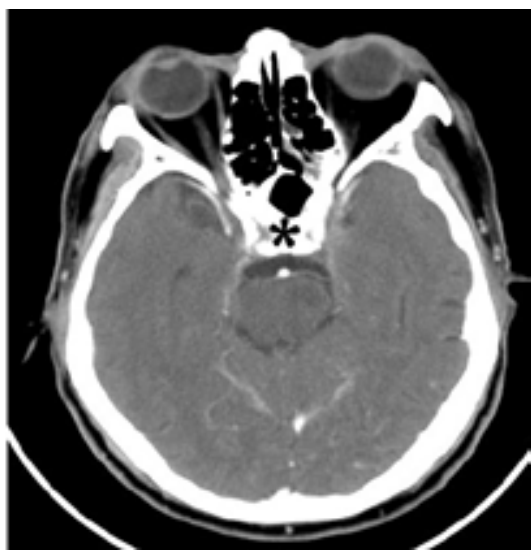

Fig. 1b. Case 1. (Two months post chemoand radiation therapy.) Axial contrastenhanced CT scan showing interval decrease in the primary nasopharyngeal mass with residual nasopharyngeal asymmetry, left more than right (asterisk). 
carcinoma of the nasopharynx. After the intravenous therapy, his vision improved to counting fingers. He was discharged with a starting dose of oral prednisolone (one $\mathrm{mg} / \mathrm{kg}$ body weight) with a gradual taper over a period of two months. By the second week of steroid therapy, his best corrected vision had improved to 6/24. At this time, the patient finally consented to radio- and chemotherapy. Three months later, after the completion of his steroid-, radio- and chemotherapy, the patient's best corrected vision in the right eye improved to 6/9. The left eye was stable, although with the limitation of abduction. A repeat CT-scan of the head reported an interval decrease in size with a residual nasopharyngeal asymmetry with the left side greater than the right (Fig. 1b).

\section{Case 2}

A 57-year-old Chinese man complained of a two to three months' history of blurred vision in the left eye. He had a history of hypertension. No other symptoms were noted.

On examination, the VA was light perception of the left eye with an afferent pupillary defect, and a slight optic disc elevation. The right eye vision and discs were normal. Motility exam and other cranial nerves were intact. An MRI of the brain revealed a large infiltrative mass at the left skull base with a thickening of the left nasopharyngeal soft tissue with significant infiltrative involvement of the surrounding structures, especially in the left orbital apex, sphenoid and ethmoid sinuses (Fig. 2a). He was immediately referred to the ENT for an excision biopsy.

While waiting for the biopsy results, he was pulsed with IV methylprednisolone one gm/day for three days and slowly tapered with oral prednisolone (starting with one $\mathrm{mg} /$ $\mathrm{kg}$ body weight) over two months. The biopsy revealed undifferentiated nasopharyngeal carcinoma. On the third day of IV steroids, his best corrected vision improved to $6 / 45$. He was discharged with an oral steroid taper starting with a dose of one $\mathrm{mg} / \mathrm{kg} / \mathrm{day}$. By the second week of the steroid therapy, his best corrected vision remained at $6 / 45$. The patient was able to identify 11 of the Ishihara color plates. At this point, his chemo- and radiation therapy started. One month later, the vision in the left eye dropped to counting fingers. The patient was now in his second session of chemotherapy. However, it was found that he had stopped his oral steroids for a month due to financial constraints. Once again he was pulsed with IV methylprednisolone one $\mathrm{gm} /$ day for three days with a gradual taper of oral steroids. However, the vision in his left eye remained at counting fingers and an optic disc pallor was noted. Two months later, even after the completion of the

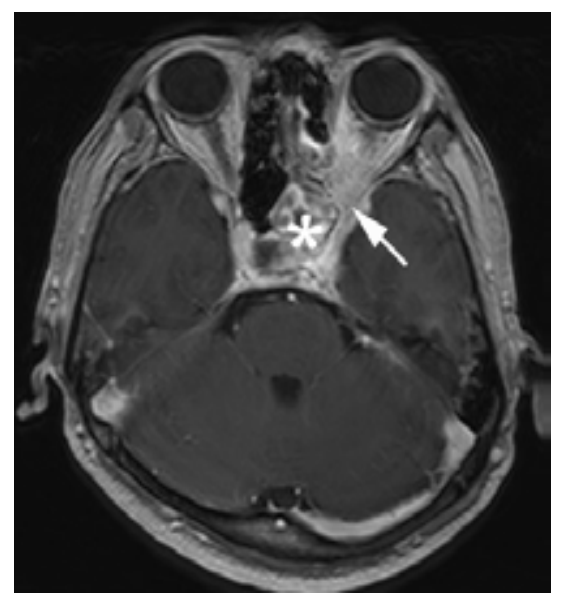

Fig. 2a. Case 2. Axial contrast-enhanced T1-weighted MRI scan of the brain showing a heterogeneously enhancing mass at the left basal skull. The extensive left ethmoid and sphenoid sinus opacification and soft tissue is suggestive of breaching and infiltration of the mass (asterisk). The infiltrative mass was noted to have significantly encroached the left orbital apex likely compressing the left optic nerve at this point (arrow). 
chemo- and radiation therapy and a positive reduction of the tumor size seen through the MRI scan with no signs of tumor progression or crowding in the area of the orbital apex (Fig. 2b), the vision in his left eye did not show any improvement.

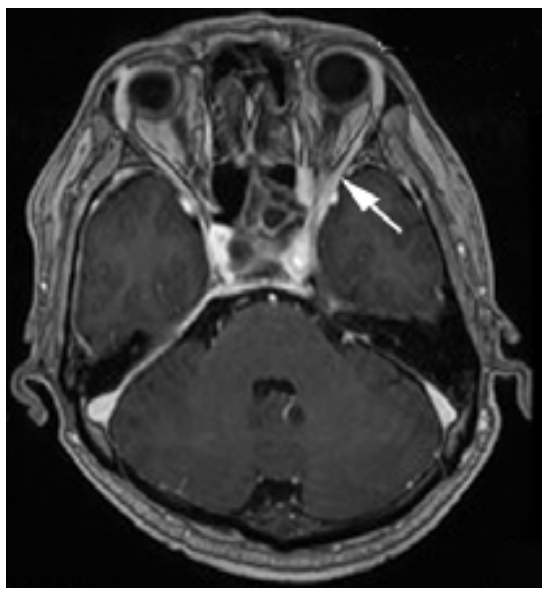

Fig. 2b. Case 2. (Two months post chemo- and radiation therapy.) Axial contrast-enhanced T1-weighted MRI scan of the brain showing that the left nasopharyngeal mass has lessened in bulkiness. There was no tumor progression or crowding of the orbital apex (arrow); its current extent was grossly similar to the prior scan.

\section{Discussion}

The ophthalmologist has two main goals when managing patients with optic neuropathy: identifying the cause of the disease and treating the visual loss. Hence, imaging becomes integral, especially in this region where the occurrence of tumors like NPC is well-documented. ${ }^{1-4}$ Pulse-steroid therapy has been known to relieve optic neuropathy that is inflammatory or compressive in nature..$^{5-7}$ In both reported cases, imaging results show infiltration in the area near the optic nerve.

It is rare for NPC to initially present as optic neuropathy. Previous reports showed that NPC patients who presented with optic neuropathy were initially diagnosed with optic neuritis, and additional improvement of vision was noted after chemo- and/or radiation therapy. ${ }^{4-7}$

Some authors theorized that methylprednisolone reduces the compressive effects of a tumor by reducing tissue edema, resulting in the immediate visual improvement of the patient. Staying on course with the appropriate chemo- and radiation therapy helps reduce the main tumor which further improves the visual function.? The debulking effect of chemo- and radiation therapy would, at the earliest, be noted one week after the initial treatment. Maintaining the patient on oral steroids while undergoing chemoand radiation therapy may provide an adjunctive effect in reducing the compressive effects of the tumor. Although some reports state that optic neuropathy can result from the paraneoplastic effect of NPC, especially in cases where imaging did not show signs of infiltration ${ }^{5}$ at the orbital apex or the areas near the optic nerve, pulse-steroid therapy can still improve vision as the steroid therapy can temporarily treat the inflammation of the paraneoplastic effect. In our two cases, the neuro-imaging studies revealed that the effect is mainly compressive due to its close proximity rather than a paraneoplastic 
effect.

In our reported cases, IV pulse methylprednisolone offered immediate visual improvement. There was a close temporal relationship between the use of steroids and visual improvement in both cases. It is the authors' preference to initially treat with IV pulse steroids over oral steroids alone. Since optic neuropathy due to NPC is very rare, the authors are unable to ascertain whether the efficacy of IV pulse steroids is better than oral steroids alone. A further study is needed to investigate this.

To optimize visual results, apart from primarily treating the NPC with the appropriate chemo- and radiation therapy, initial pulse intravenous methylprednisolone therapy followed by a gradual oral taper of steroid is paramount in controlling the inflammation/edema which is causing the optic neuropathy while stopping the steroids abruptly can lead to a recurrence of visual loss, as seen in case 2 .

IV pulse steroids with a gradual oral taper can offer immediate visual relief for patients with compressive optic neuropathy due to NPC, whilst awaiting chemo- and radiation therapy. This treatment regimen did not hinder nor compromise the primary therapeutic regimen of chemo- and radiation therapy, as seen in these two cases.

\section{Acknowledement}

Arneil U. Ang, MD, FPROS.

\section{References}

1. Hughes PJ, Scott PM, Kew J, Cheung DM, Leung SF, Ahuja AT, van Hasselt CA. Dysphagia in treated nasopharyngeal cancer. Head Neck 2000;22:393-397.

2. Witte MC, Neel III HB. Nasopharyngeal Cancer. Baltimore: Lippincott-Raven; 1998:1637-1652.

3. Park KA, Oh SY. Nasopharyngeal carcinoma presenting with rapidly progressive severe binocular optic neuropathy and periocular pain in a young man. J Neuro-Ophthalmol 2010;30:1-3.

4. Tsai CC, Ho HC, Kau HC, Kao SC, HSU WM. Optic neuritis: a rare manifestation of nasopharyngeal carcinoma. Eye 2002;16:501-503.

5. Hoh ST, Teh M, Chew SJ. Paraneoplastic optic neuropathy in nasopharyngeal carcinoma - report of a case. Singapore Med J 1991;32:170-173.

6. Prasad U, Doraisamy S. Optic nerve involvement in nasopharyngeal carcinoma. Eur J Surg Oncol 1991; 17:536-540.

7. Puri P, Puri S, Pepper I. Optic neuritis: a rare manifestation of nasopharyngeal carcinoma. Eye 2004; 18:110. 